\title{
The O*Net Jobs Classification System: A Primer for Family Researchers
}

\author{
Ann C. Crouter
}

\author{
Stephanie T. Lanza
}

Amy Pirretti

W. Benjamin Goodman

Eloise Neebe

The Family Life Project Key Investigators*

\begin{abstract}
We introduce family researchers to the Occupational Information Network, or O*Net, an electronic database on the work characteristics of over 950 occupations. The paper here is a practical primer that covers data collection, selecting occupational characteristics, coding occupations, scale creation, and construct validity, with empirical illustrations from the Family Life Project, a study of almost 1,300 families with infants born in 6 lowincome, nonmetro counties in North Carolina and Pennsylvania. We factor analyzed parents' occupations on 35 O*Net characteristics and identified 5 factors: occupational self-direction, physical hazards, physical activity, care work, and automation/repetition, variables that supplement data collected from parents directly. Applied researchers can use the $\mathrm{O}^{*} \mathrm{Net}$ to expand their knowledge of participants' work circumstances with objective data.
\end{abstract}

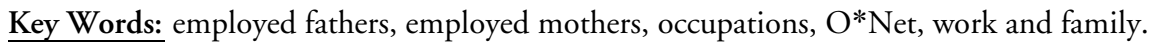

Over the past 30 years, basic and applied family researchers have become increasingly interested in the role of work in shaping the well-being of individuals, families, and communities (Perry-Jenkins, Repetti, \& Crouter, 2000). Basic researchers have moved beyond a focus on parents' employment status to study the role of occupational conditions in shaping the psychological functioning of employed adults and, in turn, family dynamics and children's development (e.g., Crouter, Bumpus, Maguire, \& McHale, 1999; Han, 2005; Parcel \& Menaghan, 1994). Applied researchers have developed workplace interventions to reduce work-family conflict (e.g., Kline \& Snow, 1994) and worked with entire communities to develop policies to support working families (e.g., Bailyn, Bookman, Harrington, \& Kochan, 2005).

One challenge that family researchers interested in work and family share is measuring the occupational conditions that are important for working parents and their families. Previous research has established that occupational conditions such as complexity and autonomy shape parents' values (Kohn \& Schooler, 1983) in ways that have implications for the quality of the environments they create at home (Parcel \& Menaghan, 1994). There is also a growing literature on how the physical, interpersonal, and emotional demands at work get carried home (see review by Perry-Jenkins et al., 2000).

Secondary data sets often do not provide detailed information about the nature of mothers' and fathers' occupations, and researchers planning new data collections often do not have sufficient time in their survey protocols to collect everything they want to know. One strategy to deal with these constraints is to supplement primary or secondary data sets with objective measures of occupational conditions from

\footnotetext{
*Ann C. Crouter is the Director of the Social Science Research Institute, 601 Oswald Tower, Penn State University, University Park, PA 16802 (ac1@psu.edu). Stephanie T. Lanza is the Scientific Director of The Methodology Center, Penn State University, 204 E Calder Way, Suite 400, State College, PA 16801 (slanza@ psu.edu). Amy Pirretti is a Research Assistant at the Department of Human Development and Family Studies, S-110 Henderson Building, Penn State University, University Park, PA 16802 (aev113@psu.edu). W. Benjamin Goodman is a Research Assistant at the Department of Human Development and Family Studies, S-110 Henderson Building, Penn State University, University Park, PA 16802 (wbg109@psu.edu). Eloise Neebe is the Director of Data Management, Campus Box 8185, The University of North Carolina, Chapel Hill, NC 27599-8185 (eloise_neebe@unc.edu). The Key Family Life Project Investigators include Lynne Vernon Feagans, Martha Cox, Clancy Blair, Peg Burchinal, Linda Burton, Keith Crnic, Nan Crouter, Patricia Garrett-Peters, Doug Granger, Mark Greenberg, Stephanie Lanza, Adele Miccio, Roger Mills-Koonce, Deborah Skinner, Cynthia Stifter, Lorraine Taylor, Emily Werner, and Mike Willoughby.
} 
other sources. The goals of this article are (a) to introduce family researchers to one such source, the Occupational Information Network, or O*Net (Peterson et al., 2001), and (b) to provide a practical primer on how to maximize the effectiveness of this tool.

Designed to replace the Dictionary of Occupational Titles (DOT; U.S. Department of Labor, 1991), the O*Net was developed by the Department of Labor to provide a continually updated electronic database on the skill requirements and characteristics of over 950 occupations that are important in the nation's economy (Peterson et al., 2001). Originally released in 1998 and updated on a regular basis, the $\mathrm{O} * \mathrm{Net}$ is available online (http:// online.onetcenter.org/) to help employees, students, employers, school guidance counselors, and job seekers make informed decisions about education, training, and work.

In early versions of the $\mathrm{O} * \mathrm{Net}$, occupations were rated by highly trained job analysts, but $\mathrm{O}^{*} \mathrm{Net}$ developers are increasingly relying on survey data from job incumbents. Using a common language and metric, the $\mathrm{O}^{*} \mathrm{Net}$ describes occupations along five work dimensions: (a) knowledge, (b) skills, (c) abilities, (d) work activities, and (e) work context. Characteristics are scored from 0 to 100 , making it easy to compare characteristics within and between occupations. Occupations are also categorized in terms of some of the personal qualities that characterize people holding these jobs including interests, work styles, and work values. In this paper, we focus on $\mathrm{O}^{*}$ Net codes for work activities and context. Like others interested in occupational socialization (e.g., Kohn \& Schooler, 1983; Parcel \& Menaghan, 1994), we see occupational dimensions as sources of influence on parents' world views and well-being that in turn may shape the opportunities they provide their children and how they interact with family members.

The $\mathrm{O}^{*}$ Net provides general information about occupations; it was not designed to provide insight on within-occupation variability. A waitress employed in a coffee shop in rural North Carolina and a waitress working in Rockefeller Center's celebrated Rainbow Room, for example, would receive identical O*Net scores, even though their day-to-day experiences would be somewhat different as a result of the nature, size, location, clientele, and management of the places where they work. The O*Net's key strength is that it provides objective estimates of a host of potentially relevant occupational characteristics. These characteristics can be used as variables in their own right or combined into multi-item scales that tap larger constructs of interest. In addition, $\mathrm{O} * \mathrm{Net}$ data can be triangulated with respondents' self-reports about their jobs to create a more detailed picture of workplace conditions.

We illustrate the utility of the O*Net here by using data from the quantitative component of the Family Life Project (FLP), a longitudinal cohort study of almost 1,300 children who were born in six lowincome, rural counties in North Carolina and Pennsylvania between September 2003 and September 2004. The study oversampled low-income families, giving the FLP a unique opportunity to examine the connections between low-income jobs, family dynamics, and child development, an important but understudied topic (Lambert, 1999). After a brief literature review that outlines how the DOT and $\mathrm{O} * \mathrm{Net}$ have been used in prior research, we provide a primer for family researchers that is organized around decisions that arise at each step of the research process, using the FLP as an example.

\section{The Research Tradition}

The promise of the $\mathrm{O} * \mathrm{Net}$ is best illustrated by reviewing how family researchers made creative use of its predecessor, the DOT. In a series of important studies conducted in the 1980s and 1990s, Menaghan and Parcel used the DOT to code the jobs of mothers (and, when applicable, their partners) who were participating in the Child-Mother portion of the National Longitudinal Surveys of Youth (NLSY; Menaghan \& Parcel, 1991, 1995; Parcel \& Menaghan, 1994). Parcel (1989) performed factor analysis on 48 items contained in the DOT to create variables on the basis of those items. The variable Menaghan and Parcel focused on in much of their subsequent research was a factor they labeled Complexity, a 19-item index that included direction, control, planning, influencing people, and low levels of repetitive or continuous processes (see Parcel \& Menaghan, 1994, p. 39).

Menaghan and Parcel's research questions built on previous research by Kohn and his colleagues (Kohn, 1977; Kohn \& Schooler, 1983) that conceptualized the workplace as a context for occupational socialization that influences workers' values and world views. Using the NLSY 1986 sample of mothers with children aged 3-6, Parcel and Menaghan 
(1994) found that, controlling for a variety of background characteristics, mothers in jobs with higher levels of complexity provided more stimulating, higher quality home environments for their young children. Moreover, longitudinal research revealed that when mothers entered low-complexity jobs the quality of home environments subsequently suffered, whereas when mothers entered jobs high in complexity the quality of home environments improved (Menaghan \& Parcel, 1995).

Although, to our knowledge, family researchers have not begun using $\mathrm{O} * \mathrm{Net}$, social scientists in other areas have begun to do so. Hadden, Kravets, and Muntaner (2004) subjected all O*Net codes to a factor analysis and identified four factors. Three of them-Substantive Complexity, People vs. Things, and Physical Demands-resembled factors other researchers had identified previously using the DOT. A fourth factor, which captured work in hierarchical organizations, was labeled Bureaucracy.

Several studies have linked O*Net codes of occupational characteristics to substance use and individual psychosocial functioning. We review this work because substance use and individual psychosocial functioning are of great interest to some family researchers (e.g., Ge, Natsuaki, \& Conger, 2006) and because this research illustrates the $\mathrm{O} * \mathrm{Net}$ 's utility in social science arenas outside the occupational domain. Zhang and Snizek (2003) coded the occupations of the participants in the 1997 National Household Survey on Drug Abuse using the O*Net. After accounting for background characteristics that might select people into jobs, occupational characteristics such as autonomy and workload had little direct association with alcohol and drug use. Cocaine use, however, was higher among people whose jobs offered little variety. Zimmerman, Christakis, and Stoep (2004) linked the 1992 wave of the NLSY 1979 cohort to the O*Net to explore how young adults' depressive symptoms were linked to their occupational characteristics. They focused on eight work attributes: Recognition (or status), Opposition, Security, Machine Pace, Sociability, Morality (whether the job exposes the person to moral choices), Physically Uncomfortable, and High Compensation. Controlling for demographic characteristics, low recognition or status was highly related to higher depressive symptoms for young men and for young Black women. In addition, young women and Latino men in physically uncomfortable jobs reported higher levels of depressive symptoms. Zimmerman et al. acknowledged that it is unclear whether occupational characteristics affected depression or whether depressed people gravitated to (or failed to leave) occupations with negative qualities, but their research illustrates a primary asset of using $\mathrm{O} * \mathrm{Net}$-derived variables: Individuals reported only on depressive symptoms, not work situations, thereby eliminating shared method variance as a source of inflated associations.

We organized the remainder of this paper as a practical guide for family researchers who are interested in integrating objective measures of occupations into their research. We organize this primer around the various steps that research entails: data collection, choosing $\mathrm{O}^{*} \mathrm{Net}$ characteristics, data reduction and scale creation, and checking construct reliability and validity. Where applicable, we include special considerations for longitudinal research. To illustrate these steps, we discuss decisions we have made in the context of the FLP. The purpose of this article is to showcase the potential utility of the $\mathrm{O}^{*} \mathrm{Net}$ for family researchers, not to present original research per se; however, before presenting the primer, we provide a brief introduction to the FLP sample and methods to put our $\mathrm{O} *$ Net-related decisions in context.

\section{FLP: Sample and Methods}

The FLP design involved intensive data collection via home and childcare visits when children were approximately 2, 6, 15, 24, and 35 months of age. At each wave, data were collected via interviews with mothers and, where possible, secondary caregivers (e.g., mother's partner, baby's grandmother); observations of parent-child interaction; collection of biomarker data; and infant and child assessments.

\section{Participants}

The FLP sample is representative of families with newborns in six low-income, nonmetro counties in North Carolina and Pennsylvania. A hospital in each county was chosen (at random, with probability proportional to size, when there was more than one), and each day of the recruitment year FLP staff visited all new mothers who had given birth the day before. They provided a pamphlet describing the project, answered questions, asked screening questions related to income, language use, and race, and requested contact information and permission to contact families several weeks later about 
participation. Families were considered eligible if they spoke English as the primary language at home, did not intend to move out of the state in the next 3 years, and had parental rights. Families that did not provide screening information could not be recruited into the study. Screening data were sent to the data management core at the University of North Carolina, which in turn randomly selected families and notified sites about whom to recruit. African American families and families living at or below $200 \%$ of poverty were oversampled because the project was focused on the interplay of race, geographic isolation, and poverty in shaping pathways to competence for children in nonmetro environments. Hospital recruiters identified 5,471 women who resided in target counties and who gave birth to a child in the 12-month recruitment period. A total of $1,515(28 \%)$ of these families were ineligible, leaving the population of eligible families at 3,956. Of these, 2,691 (68\%) were willing to be considered for participation, 1,571 (58\%) of them were selected into the study according to the study design, and $1,292(82 \%)$ were successfully enrolled in the study.

At the initial data collection point (when children were about 2 months of age), 1,292 families participated (773 in North Carolina and 519 in Pennsylvania). We relied on the 6-month data because we were interested in employment, and many mothers were not working at the 2 -month visit. At 6 months, 1,204 families participated, an overall retention rate of $93 \%$. In those 1,204 families, 619 mothers or mother figures were employed at least $5 \mathrm{hr}$ per week. Of those, 613 were the biological mother of the target infant. Of the 613 biological mothers, we were able to code jobs for 603 (some jobs are so unusual that they do not fit $\mathrm{O}^{*} \mathrm{Net}$ codes). Of the secondary caregivers, 625 were employed at least $5 \mathrm{hr}$ per week, and of these, 552 were mother's partner (most, but not all, were the biological father of the target child). Of these, 534 had jobs that could be coded with the O*Net. Of the 603 mothers, 302 were living with employed partners for whom we had $\mathrm{O}^{*} \mathrm{Net}$ codes. Thus, we have $\mathrm{O}^{*} \mathrm{Net}$ data for both partners in 302 dual-earner families.

\section{Procedures}

When infants were approximately 6 months of age, data collection staff made two home visits, about a week apart. The visits involved an array of different kinds of data collection including interviews with mothers and, where applicable, secondary caregivers. The interviews were administered on laptop computers and included questions about jobs and occupational conditions. Relevant details on job measures from the interviews appear in the primer below.

\section{An 0*Net Primer for Family Researchers}

\section{Data Collection}

Researchers collecting their own data must ask about respondents' occupations in sufficient detail that they can be subsequently coded. Interested in a thorough picture of mothers' and secondary caregivers' work situations, the FLP created a jobs grid that asked a series of questions about each job at which respondents spent $5 \mathrm{hr}$ or more per week, including the job title, employer, and a short description of primary activities and duties. We trained data collection staff to ask for details. It was insufficient, for example, to record that someone was a "teacher" because the $\mathrm{O} * \mathrm{Net}$ has 78 separate codes for teachers. Instead, we asked home visitors to provide specific job titles such as "high school English teacher," "nursing home nurse's aide," or "fitness instructor at the Y." Knowing the employer and some of the tasks involved in the job helped us to choose between similar occupation codes.

We defined the job in which the person spent the most time per week as the primary job. FLP respondents completed a variety of self-report questionnaires about the primary job, and we coded all primary jobs with the $\mathrm{O} * \mathrm{Net}$, as will be described below. We also collected job titles, employer information, and job tasks and activities for all other jobs so that we could later code them if we chose.

Because we collected interview data using preprogrammed laptop computers, interview questions and related skip patterns could be preprogrammed into a shell that was updated for each family using information from the previous wave of data collection. Thus, at the 6-month visit, when the interviewer came to the section of the interview about jobs, the jobs grid information completed at the 2month visit popped up. The interviewer then asked whether the person still held each job and whether or not he or she had added any new jobs. For jobs 
that had not changed, we retained the same O*Net codes, but we asked again about work hours, shift, wages, and other work circumstances that may have changed. We asked for complete information about new jobs so that they could be coded later.

\section{Selecting O*Net Characteristics of Interest}

The O*Net includes several hundred occupational characteristics. Thus, researchers must specify the aspects of work they wish to measure and then map the relevant occupational characteristics onto those constructs. Zimmerman et al. (2004), for example, chose work constructs that the literature suggested might underlie depressive symptoms. In our case, we identified seven general, conceptually interrelated domains that we were interested in because the literature suggested they might have a bearing on how parents felt about themselves, how they structured their home environments, or how they treated their young children. Three of our constructs mapped on to Kohn and Schooler's (Kohn, 1977; Kohn \& Schooler, 1983) notion of occupational self-directioncomplexity, supervision, and routinization, constructs that were also important in Parcel and Menaghan's (1994) research. Three constructs pertained to different kinds of stressors people might encounter at work: interpersonal stress (e.g., customer service), physical hazards (e.g., exposure to extreme temperatures), and the kind of stress entailed in taking care for others (i.e., care work). The final construct was physical activity. Here, we reasoned that adults in physically active jobs might be tired and depleted at the end of the day and therefore perhaps less available to interact responsively with children. Having identified these conceptual domains, we sifted through the $\mathrm{O} * \mathrm{Net}$ characteristics and identified 35 characteristics of interest (see Table 1). Our next step was to identify the most efficient means of carrying out the coding itself.

\section{Coding Guidelines}

Because the $\mathrm{O}^{*} \mathrm{Net}$ is online, the most obvious strategy is to have coders go online to extract the values for each characteristic of interest for each occupation. This was our initial approach, but we do not recommend it. Even careful coders make coding errors. More importantly, the $\mathrm{O} * \mathrm{Net}$ is constantly being updated. FLP families were recruited over the course of a full year. Thus, it took 12 months to complete a wave of data collection and, hence, to code all employed adults' jobs at any one data point because coders were expected to keep pace with data collection. Major O*Net updates occur twice a year, and minor updates may occur at any time. If we had relied on the online system, two FLP respondents with the same occupation, participating in the same wave of data collection, might have had different values on some occupational characteristics because one person's data were coded before an update occurred and the other person's were coded after the update took place.

A more efficient approach that reduces human error and bypasses $\mathrm{O}^{*} \mathrm{Net}$ updating is to download the most recent version of $\mathrm{O}^{*} \mathrm{Net}$ (these are free and can be found in the O*Net Resource Center: http:// www.onetcenter.org/). We used Version 6.0, for example, to code the 6-month data. Note, however, that the various $\mathrm{O}^{*} \mathrm{Net}$ versions are continually updated and revised until they are "retired." Choosing the most recently retired database ensures that the codes, at least for that version, are in final form. The coder identifies the appropriate $\mathrm{O} * \mathrm{Net}$ occupation code (e.g., the code for waiters and waitresses is 35-3031.00), and the values for the occupational characteristics in the database are electronically merged to the occupation codes in the sample (see Appendix for more details). This means that all nurses' aides, waitresses, or middle school special education teachers receive the same scores regardless of when their jobs were coded. Making an accurate match between a respondent's description of his or her job and the $\mathrm{O}^{*}$ Net occupation code is thus the critical coding activity. We checked reliability by having two coders code a random sample of 137 unique jobs in our data set. Intercoder reliability was satisfactory; Pearson correlation coefficients ranged from .80 to .92 for the five scales we created (see Data Reduction and Scale Creation).

Additional issues arise when conducting longitudinal research. For example, we used Version 6 to code occupations for the 6-month visits, but part way through the 15-month visits, Version 7 became available. Fortunately, if one downloads each version as it becomes available, researchers can later decide which version of the $\mathrm{O} * \mathrm{Net}$ to apply in a given analysis. Thus, for cross-sectional analysis of, say, the 15-month data, we would choose Version 7 because it was the most up-to-date data set at the time that those data were collected. For example, in several years, however, when we will have occupational data 
Table 1. Summary of Exploratory Factor Analysis Results for $O^{*}$ Net Job Characteristics Using Varimax Rotation $(n=380$ unique jobs)

\begin{tabular}{|c|c|c|c|c|c|}
\hline \multirow[b]{2}{*}{ Item } & \multicolumn{5}{|c|}{ Factor Loadings } \\
\hline & Self-Direction & $\begin{array}{l}\text { Physically } \\
\text { Hazardous }\end{array}$ & $\begin{array}{l}\text { Physically } \\
\text { Active }\end{array}$ & $\begin{array}{l}\text { Care } \\
\text { Work }\end{array}$ & $\begin{array}{c}\text { Automation/ } \\
\text { Repetition }\end{array}$ \\
\hline Organize, plan, prioritize & .85 & -.13 & -.20 & .11 & -.03 \\
\hline Think creatively & .74 & -.11 & -.15 & .10 & -.07 \\
\hline Make decisions, solve problems & .78 & .01 & -.30 & .22 & -.09 \\
\hline Develop objectives, strategies & .87 & -.09 & -.16 & .13 & -.18 \\
\hline Schedule work, activities & .89 & -.12 & -.08 & .10 & -.08 \\
\hline Responsible for outcomes, results & .83 & .10 & .02 & .12 & .27 \\
\hline Coordinate work, activities & .91 & -.03 & .02 & .01 & -.03 \\
\hline Guide, direct, motivate others & .92 & -.01 & .03 & - & -.02 \\
\hline Coordinate, lead others & .88 & -.03 & -.04 & .24 & .11 \\
\hline Frequency of conflict situations & .67 & -.12 & -.13 & .54 & .16 \\
\hline Resolve conflict, negotiate & .79 & -.22 & -.12 & .33 & .08 \\
\hline Establish interpersonal relationships & .74 & -.33 & -.14 & .38 & -.03 \\
\hline Train, teach others & .82 & -.14 & .03 & .22 & -.14 \\
\hline Provide consultation, advice & .74 & -.19 & -.27 & .09 & -.27 \\
\hline Coach, develop others & .88 & -.14 & - & .20 & -.08 \\
\hline Develop, build teams & .89 & -.07 & - & .24 & .09 \\
\hline Exposed to hazardous conditions & -.11 & .84 & .16 & - & .05 \\
\hline Exposed to hazardous equipment & -.23 & .81 & .16 & -.21 & .19 \\
\hline Exposed to contaminants & -.02 & .80 & .27 & .16 & .26 \\
\hline Sounds, noise levels are uncomfortable & .11 & .69 & .12 & .19 & .50 \\
\hline Very hot or cold temperatures & -.06 & .64 & .45 & -.04 & .07 \\
\hline $\begin{array}{l}\text { Spend time climbing ladders, } \\
\text { scaffolds, poles }\end{array}$ & -.17 & .61 & .32 & -.24 & -.14 \\
\hline Perform general physical activities & -.27 & .47 & .68 & .05 & -.03 \\
\hline Spend time walking, running & .14 & .09 & .79 & .12 & - \\
\hline Spend time bending, twisting body & -.23 & .52 & .66 & .06 & .15 \\
\hline Spend time standing & -.20 & .21 & .84 & -.07 & -.03 \\
\hline Spend time sitting & .12 & -.28 & -.85 & -.03 & -.06 \\
\hline Assist, care for others & .42 & -.20 & .04 & .72 & -.12 \\
\hline Deal with physically aggressive people & .31 & .02 & .04 & .80 & .01 \\
\hline Deal with unpleasant, angry people & .55 & -.22 & -.05 & .65 & .21 \\
\hline Exposed to disease or infections & .16 & .16 & .13 & .78 & -.03 \\
\hline Importance of repeating same tasks & .14 & .03 & -.08 & .24 & .84 \\
\hline Pace determined by equipment speed & -.28 & .46 & .01 & -.27 & .56 \\
\hline Spend time making repetitive motions & -.19 & .14 & .26 & -.04 & .79 \\
\hline Degree of automation & -.05 & .20 & -.40 & -.23 & .60 \\
\hline Eigenvalues & 14.82 & 6.23 & 3.00 & 2.18 & 1.22 \\
\hline $\begin{array}{l}\% \text { of variance explained by factor } \\
\text { Cronbach's alpha: }\end{array}$ & 12.10 & 4.84 & 4.30 & 3.45 & 2.77 \\
\hline Mothers $(n=603)$ & .96 & .81 & .89 & .84 & .82 \\
\hline Secondary caregivers $(n=534)$ & .98 & .90 & .89 & .86 & .68 \\
\hline
\end{tabular}


from mothers and secondary caregivers when children were $6,15,24$, and 35 months of age, we will want to analyze trajectories of occupational conditions to investigate whether increased self-direction on the job predicts increased parental stimulation of children's learning at home. For longitudinal analyses, it would be important that all waves of occupations are coded using the same version of $\mathrm{O}^{*} \mathrm{Net}$. We will choose the most up-to-date version and back-code all waves of data with that version electronically because doing so takes advantage of the most current information available on $\mathrm{O} * \mathrm{Net}$ occupations.

\section{Data Reduction and Scale Creation}

Having identified the 35 characteristics of interest and coded occupations, we next sought to create scales with good psychometric properties using factor analysis. Because the constructs of interest were interrelated conceptually, and a factor analysis had not been done on them before, we took an exploratory approach. We used the occupation, not the person, as the unit of analysis because some occupations were quite common and we wanted to avoid common occupations having disproportionate influence on the factor solution. Indeed, the most common occupations for mothers were cashiers $(n=43)$ and nursing aides, orderlies, and attendants $(n=32)$ and, for secondary caregivers, construction carpenters $(n=18)$ and automotive master mechanics $(n=12)$. We decided to combine mothers' and secondary caregivers' occupations in the same analysis ( $n=380$ unique occupations) because the O*Net codes are gender neutral and, although we realized that strong forces operate to select men and women into different jobs (Reskin, 1993), we had no reason to believe that occupational characteristics would load on to different constructs for men and women. Indeed, we replicated the factor analysis, allowing common jobs to be represented as often as they appeared in the sample, and the results were virtually identical. Similarly, we replicated the factor analysis separately on mothers and on secondary caregivers, and again, the findings were very similar.

To reduce our data, we conducted an exploratory factor analysis with an orthogonal rotation (i.e., Varimax). As can be seen in Table 1, the analysis revealed a fairly simple, five-factor structure in which every item loaded onto one and only one factor. The items that defined each factor were the same regardless of whether an orthogonal solution or an oblique solution was used. Consistent with Tabachnick and Fidell's (2001) criteria for "very good" factor loadings, we considered items with a loading of $>.55$ to be measuring that construct. We labeled the first factor self-direction to signal its similarity to Kohn and Schooler's (1983) selfdirection construct. The greatest number of items $(n=16)$ loaded on this factor. It included items we had conceptualized as representing complexity (e.g., "making decisions, solving problems"; "thinking creatively") as well as some items that we thought might reflect interpersonal stress (e.g., "resolving conflict and negotiating"), because these interpersonal processes come bundled with jobs that are more complex and self-directed. We labeled the second factor hazardous physical conditions because the 6 items all pertained to hazards, contaminants, or extremes of noise or temperature. The 5 items that made up the third factor all pertained to physical activity such as running, bending, or standing. The fourth factor was a variant on our idea of care work. The four items addressed caring for others and dealing with unpleasant or aggressive people. Interestingly, the highest loading item was "exposed to disease or infection" suggesting that this constellation of care work activities often pertains to caring for the sick. The fifth factor, automation/repetition, included four items that signaled automated, repetitive work in which pace is determined by the equipment, not the operator.

To create scale scores, we took the mean of the items that had loaded on each factor (bolded values in Table 1). As noted by Comrey and Lee (1992), this approach is acceptable for exploratory studies given the fairly simple factor structure as well as the high degree of similarity in variance across items. Cronbach's alphas indicated satisfactory internal consistency for all five scales (see bottom of Table 1). Table 2 presents the occupations in the FLP sample that emerged as having the highest and lowest values on the five $\mathrm{O}^{*} \mathrm{Net}$ scales. Seeing the actual occupations behind the constructs helps them come to life. Mothers with the highest exposure to hazardous conditions, for example, were package/filling machine operators, fire fighters, and emergency medical technicians/paramedics.

The shaded quadrants in Table 3 provide information about the associations among the $\mathrm{O} * \mathrm{Net}$ variables for mothers and partners. For mothers, self-direction was associated only with care work; 
Table 2. Occupations for Mothers and Partners Scoring Highest and Lowest on $O^{*}$ Net Factors

\begin{tabular}{|c|c|c|}
\hline & Mother & Partner \\
\hline \multicolumn{3}{|c|}{ Self-direction } \\
\hline \multirow[t]{3}{*}{ Top occupations } & Coaches and scouts & Coaches and scouts \\
\hline & Advertising managers & Training and development managers \\
\hline & Medical/health services managers & Private sector executives \\
\hline \multirow[t]{3}{*}{ Lowest occupations } & Tire builders & Tire builders \\
\hline & Hand pressers & Floor sander and finishers \\
\hline & Hand cutter/trimmers & Production laborers \\
\hline \multirow{4}{*}{ Top occupations } & Hazardous physical conditions & \\
\hline & Package/filling machine operators & Heating/air conditioning mechanics \\
\hline & Fire fighters & Bus/truck/diesel mechanics \\
\hline & Emergency medical technicians/paramedics & Heavy equipment mechanics \\
\hline \multirow[t]{3}{*}{ Lowest occupations } & Claims examiners & Educational counselors \\
\hline & Tax examiners & Central office operators \\
\hline & Educational counselors & Medical sales representatives \\
\hline \multicolumn{3}{|c|}{ Physical activity } \\
\hline \multirow[t]{3}{*}{ Top occupations } & Construction carpenters & Radiology technicians \\
\hline & Waiters/waitresses & Carpet installers \\
\hline & Farm workers & Electricians \\
\hline \multirow[t]{3}{*}{ Lowest occupations } & Data entry keyers & Public relations specialists \\
\hline & Graphic designers & Graphic designers \\
\hline & Personnel recruiters & Central office operators \\
\hline \multicolumn{3}{|c|}{ Care work } \\
\hline \multirow[t]{3}{*}{ Top occupations } & Correctional officers & Correctional officers \\
\hline & Probation officers & Psychiatric aides \\
\hline & Psychiatric aides & Licensed practical nurse \\
\hline \multirow[t]{3}{*}{ Lowest occupations } & Numerical tool/process control programmers & Cabinet makers/bench carpenters \\
\hline & Molding and casting workers & Tree trimmers \\
\hline & Data entry keyers & Washing equipment operators \\
\hline \multirow{4}{*}{ Top occupations } & Automation/repetition & \\
\hline & Medical and clinical lab technologists & Dispatchers \\
\hline & Package/filling machine operators & Machine feeders \\
\hline & Cashiers & Package/filling machine operators \\
\hline \multirow[t]{3}{*}{ Lowest occupations } & Psychology teachers, postsecondary & Clergy \\
\hline & Social and human service assistants & Psychology teachers, postsecondary \\
\hline & Medical sales representatives & Funeral directors \\
\hline
\end{tabular}

jobs high on care work tended also to be high on self-direction. For partners, self-direction was positively associated with care work as well, but in addition, partners in self-directed jobs were significantly less exposed to hazardous conditions, physical activity, and automation. For both mothers and partners, working in hazardous conditions was positively associated with physical activity and automation. Physical activity was positively correlated with care work and automation for mothers and with automation for partners.

The bottom left-hand quadrant of Table 3 summarizes within-couple associations for the 302 pairs for whom we have $\mathrm{O}^{*} \mathrm{Net}$ data on both members of the dyad. The shaded diagonal within that quadrant draws attention to the within-couple correlations for the same $\mathrm{O}^{*} \mathrm{Net}$ factors. Mothers in jobs with higher self-direction, physical activity, and automation shared 
Table 3. Intercorrelations Among Mothers' $(M)$ and Partners' $(P) O^{*} N e t$ Scores ( $n=602$ mothers, 534 partners, and 302 mother-partner dyads)

\begin{tabular}{|c|c|c|c|c|c|c|c|c|c|c|}
\hline Variables & 1 & 2 & 3 & 4 & 5 & 6 & 7 & 8 & 9 & 10 \\
\hline 1. M Self-direction & - & & & & & & & & & \\
\hline $\begin{array}{l}\text { 2. M Hazardous } \\
\text { conditions }\end{array}$ & .07 & - & & & & & & & & \\
\hline 3. M Physical activity & -.07 & $.49 * * *$ & - & & & & & & & \\
\hline 4. M Care work & $.45 * * *$ & .04 & $.29 * * *$ & - & & & & & & \\
\hline $\begin{array}{l}\text { 5. M Automation/ } \\
\text { repetition }\end{array}$ & -.01 & $.40 * * *$ & $.17 * * *$ & -.07 & - & & & & & \\
\hline 6. P Self-direction & $.21 * * *$ & .02 & $-.20 * * *$ & -.01 & -.03 & - & & & & \\
\hline $\begin{array}{l}\text { 7. P Hazardous } \\
\text { conditions }\end{array}$ & -.06 & -.02 & $.14 *$ & .09 & .10 & $-.11^{*}$ & - & & & \\
\hline 8. P Physical activity & $-.15 * *$ & .06 & $.22 * * *$ & .03 & $.13^{*}$ & $-.24 * * *$ & $.62 * * *$ & - & & \\
\hline 9. P Care work & .10 & $.14^{*}$ & -.11 & .07 & -.03 & $.61 * * *$ & $-.10^{*}$ & -.08 & - & \\
\hline 10. P Automation/ & -.06 & .10 & .06 & .03 & $.24 * * *$ & $-.11^{*}$ & $.41 * * *$ & $.19 * * *$ & -.02 & - \\
\hline
\end{tabular}
repetition

Note: $\mathrm{M}=$ mother, $\mathrm{P}=$ partner. The upper right quadrant provides information about the associations among the $\mathrm{O}^{*} \mathrm{Net}$ variables for mothers. The lower left quadrant provides that information about fathers. The shaded diagonal draws attention to the within-couple correlations for the same $\mathrm{O} * \mathrm{Net}$ variables.

$* p<.05 . * * p<.01 . * * * p<.001$.

lives with partners who tended to share those job characteristics. These within-couple associations may reflect assortative mating; to the extent that individuals choose partners with similar levels of education and training, it is not surprising that there are matches on some occupational circumstances.

\section{Checking Construct Validity}

To explore the validity of the O*Net scales, we examined associations with background characteristics that represented possible selection effects into jobs (e.g., education, literacy, age) and self-report data on job characteristics (e.g., wages, self-reported occupational self-direction, prestige, and shift work). For the sake of brevity, we present data only on mothers; findings for partners were very similar.

Beginning with possible selection effects, as one would expect, better educated mothers held jobs higher in self-direction $(r=.40, p<.001)$ and care work $(r=.15, p<.001)$ and lower in physical activity $(r=-.32, p<.001)$, hazardous conditions $(r=-.12, p<.01)$, and automation/repetition $(r=-.27, p<.001)$. Using the K-Fast screening instrument (Kaufman \& Kaufman, 1994), we found that mothers with better reading skills held jobs that were higher in self-direction $(r=.23, p<.001)$ and lower in physical activity $(r=-.30, p<.001)$ and automation $(r=-.15, p<.001)$. As one might expect, given the time it takes to acquire training, older mothers held jobs that were higher on self-direction $(r=.26, p<.001)$ and lower on physical activity $(r=-.32, \mathrm{p}<.001)$ and automation/repetition $(r=-.16, p<.001)$.

Examination of associations between the $\mathrm{O} * \mathrm{Net}$ scales and mothers' self-reported work characteristics provides further evidence of validity. Mothers who earned higher wages held jobs involving higher selfdirection $(r=.26, p<.001)$ and less physical activity $(r=-.29, p<.001)$ and automation/repetition $(r=-.17, p<.001)$. During the 6-month home visit, mothers completed an 11-item adaptation of Lennon's measure of Occupational Self-Direction (Lennon, 1994, Cronbach's $\alpha=.86$; sample item: "You decide on your own how to go about doing the work"). Scores on the self-report self-direction measure were correlated positively with the $\mathrm{O}^{*} \mathrm{Net}$ measure of self-direction $(r=.33, p<.001)$ and negatively with physical activity $(r=-.25, p<$ $.001)$. Jobs were also coded for occupational prestige using the National Opinion Research Center (NORC) coding system (Nakao \& Treas, 1994). Higher prestige jobs were characterized by higher self-direction $(r=.46, p<.001)$ and care work $(r=.34, p<.001)$ and lower physical activity $(r=$ $-.42, p<.001)$, exposure to hazardous conditions $(r=-.17, p<.001)$, and automation $(r=-.35$, $p<.001)$. 
We conducted one-way analyses of variance to examine the associations between the $\mathrm{O}^{*} \mathrm{Net}$ factors and mothers' work shifts (a five-level factor: evening, rotating, day, irregular, and night; "other" was excluded from this analysis). Because cell sizes were uneven, we examined Type III sums of squares. Significant findings were followed up with Tukey tests. Mothers' shift was significantly related to hazardous conditions, $F(4,576)=11.99, p<.001$. As can be seen in Table 4, mothers who worked a day shift were less exposed to hazardous conditions than those on all other shifts, and those on rotating shift were significantly more exposed to hazards than those on day or evening shifts. A significant effect for shift on physical activity, $F(5,576)=26.12, p<.001$, revealed that day shift jobs were lower on physical activity than all other shifts. Similarly, jobs on day shift were characterized by less automation/repetition than jobs with irregular or evening shifts, $F(4$, $576)=7.57, p<.001$. In contrast, care work described night shift jobs; care work was higher on night shift than all other shifts, $F(5,576)=3.93$, $p<.01$. Shift work was not significantly associated with self-direction.

Having completed these steps, the FLP has a set of objective measures of jobs that can be used in subsequent analyses about the implications of occupational conditions for parenting and, in turn, child development. As the project moves through time, we will replicate the factor analysis to make sure that the changing mix of employed caregivers and jobs does not affect the factors. Because the sample included such a wide range of jobs at the 6-month visit, however, we do not expect the factors to change.

\section{Conclusions}

It is our hope that this primer will stimulate new research on occupational conditions and their implications for families. Each research team will want to tailor its approach to the $\mathrm{O}^{*} \mathrm{Net}$ on the basis of its research questions and the nature of the population of interest, but the decision points highlighted in this brief article should be widely applicable. For family researchers, the key strengths of the $\mathrm{O} * \mathrm{Net}$ include the care that has gone into establishing the classification system and creating and updating the codes, the diversity of occupational characteristics that are assessed, and the fact that these data are objective and, thus, enable researchers to limit relying on self-report data. Furthermore, the $\mathrm{O} * \mathrm{Net}$ is free and easily accessible. Although researchers need to remember that the $\mathrm{O}^{*} \mathrm{Net}$ is continually being updated and plan accordingly, the updating is an

Table 4. Means (and standard deviations) of $O^{*}$ Net Factor Scores as a Function of Maternal Work Shift $(n=581)$

\begin{tabular}{|c|c|c|c|c|c|}
\hline \multirow[b]{2}{*}{ O*Net Factors } & \multicolumn{5}{|c|}{ Maternal Work Shift } \\
\hline & Day & Evening & Night & Rotating & Irregular \\
\hline \multicolumn{6}{|l|}{ Self-direction } \\
\hline$M$ & $41.74 a$ & $38.68_{a}$ & $43.98_{a}$ & $37.25 \mathrm{a}$ & $41.30_{\mathrm{a}}$ \\
\hline$S D$ & 17.64 & 13.62 & 19.14 & 19.95 & 13.94 \\
\hline \multicolumn{6}{|c|}{ Hazardous condition } \\
\hline$M$ & $16.72_{\mathrm{a}}$ & $21.23_{\mathrm{b}}$ & $23.64_{b, c}$ & $26.95_{c}$ & $23.67_{b, c}$ \\
\hline$S D$ & 10.98 & 9.57 & 15.27 & 16.87 & 10.23 \\
\hline \multicolumn{6}{|l|}{ Physical activity } \\
\hline$M$ & $46.31_{\mathrm{a}}$ & $60.35 b$ & $56.41_{b}$ & $56.90_{\mathrm{b}}$ & $62.94_{b}$ \\
\hline$S D$ & $17.69^{\circ}$ & 14.55 & 12.65 & 14.23 & 11.70 \\
\hline \multicolumn{6}{|l|}{ Care work } \\
\hline$M$ & $37.51_{\mathrm{a}}$ & $39.19_{a}$ & $54.30_{\mathrm{b}}$ & $39.62_{a}$ & $41.13_{\mathrm{a}}$ \\
\hline$S D$ & 20.28 & 17.79 & 23.82 & 26.22 & 16.78 \\
\hline \multicolumn{6}{|c|}{ Automation/repetition } \\
\hline$M$ & $30.92_{\mathrm{a}}$ & $38.17_{b}$ & $32.67_{\mathrm{a}, \mathrm{b}}$ & $33.45_{\mathrm{a}, \mathrm{b}}$ & $38.38_{\mathrm{b}}$ \\
\hline$S D$ & 14.78 & 14.75 & 12.77 & 8.17 & 13.21 \\
\hline
\end{tabular}


important advantage because it mirrors changes that are continually taking place in the workplace as a function of technology and innovation. Indeed, the DOT became obsolete precisely because, with its cumbersome, nonelectronic format, developers could not keep up with the rapidly changing world of work.

Although we have focused on the utility of the $\mathrm{O}^{*} \mathrm{Net}$ in basic, longitudinal family research, the $\mathrm{O}^{*} \mathrm{Net}$ is also potentially useful in intervention and policy research. For example, Schock, Gavazzi, Fristad, and Goldberg-Arnold (2002) reported that fathers cited work schedules and work demands as obstacles to participating fully in a program designed to address children's mood disorders. A deeper knowledge of fathers' occupational characteristics might provide insight into the dynamics underlying program participation and help interventionists design programs to meet their needs. The $\mathrm{O}^{*}$ Net could also be an effective tool in research examining the transition from welfare to work. In concluding a provocative article showing that the quality of young children's home environments actually declined when mothers entered jobs low in wages and complexity, Menaghan and Parcel (1995) speculated that policies encouraging mothers to move from welfare to work might have negative effects on families and children if mothers encountered negative occupational conditions on the job. The $\mathrm{O}^{*}$ Net provides researchers interested in welfare policy with a wide range of possible occupational variables that could be used to characterize employed mothers' jobs.

Family practitioners may also find the $\mathrm{O}^{*} \mathrm{Net}$ useful when faced with cases in which a parent's work emerges as a family issue. To learn more about what the parent may be experiencing at work, the practitioner would simply go online, identify the most relevant occupation code associated with the job in question, and read the summary of the characteristics of that job. Clicking "details" would reveal the characteristics in each domain (e.g., activities, context), arrayed in numerical order from most to least salient, providing an immediate impression of the conditions most relevant to incumbents.

A final caveat: $\mathrm{O}^{*} \mathrm{Net}$ measures, even when carefully constructed, are not substitutes for self-report data; they tap general properties of occupations, not the specific experiences individuals encounter performing a particular job in a specific community, organizational, and interpersonal context. The promise of the $\mathrm{O}^{*} \mathrm{Net}$ for family research lies in the creative triangulation of objective occupation data with more subjective assessments of jobs.

\section{References}

Bailyn, L., Bookman, A., Harrington, M., \& Kochan, T. A. (2005). Workfamily interventions and experiments: Workplaces, communities, and society (Working Paper No. WPC0021). Cambridge, MA: Massachusetts Institute of Technology, MIT Workplace Center.

Comrey, A. L., \& Lee, H. B. (1992). A first course in factor analysis (2nd ed.). Hillsdale, NJ: Lawrence Erlbaum.

Crouter, A. C., Bumpus, M. F., Maguire, M. C., \& McHale, S. M. (1999). Linking parents' work pressure and adolescents' well-being: Insights into dynamics in dual-earner families. Development Psychology, 35, $1453-1461$.

Ge, X., Natsuaki, M. N., \& Conger, R. D. (2006). Trajectories of depressive symptoms and stressful life events among male and female adolescents in divorced and nondivorced families. Development and Psychopathology, 18, 253-273.

Hadden, W. C., Kravets, N., \& Muntaner, C. (2004). Descriptive dimensions of US occupations with data from the O*Net. Social Science Research, 33, 64-78.

Han, W. (2005). Maternal nonstandard work schedules and child cognitive outcomes. Child Development, 76, 137-154.

Kaufman, A. S., \& Kaufman, N. L. (1994). Kaufman Functional Academic Skills Test (K-FAST). Circle Pines, MN: American Guidance Service.

Kline, M. L., \& Snow, D. L. (1994). Effects of a worksite coping skills intervention on the stress, social support, and health outcomes of working mothers. Journal of Primary Prevention, 15, 105-121.

Kohn, M. L. (1977). Class and conformity: A study in values (2nd ed.). Chicago: University of Chicago Press.

Kohn, M. L., \& Schooler, C. (1983). Work and personality: An inquiry into the impact of social stratification. Norwood, NJ: Ablex.

Lambert, S. J. (1999). Lower-wage workers and the new realities of work and family. The Annals of the American Academy of Political and Social Science, 562, 174-190.

Lennon, M. C. (1994). Women, work, and well-being. Journal of Health and Social Behavior, 35, 235-247.

Menaghan, E. G., \& Parcel, T. L. (1991). Determining children's home environments: The impact of maternal characteristics and current occupational and family conditions. Journal of Marriage and the Family, 53, 417-431.

Menaghan, E. G., \& Parcel, T. L. (1995). Social sources of change in children's home environments: The effects of parental occupational experiences and family conditions. Journal of Marriage and the Family, $57,69-84$.

Nakao, K., \& Treas, J. (1994). Updating occupational prestige and socioeconomic scores: How the new measures measure up. Sociological Methodology, 24, 1-72.

Parcel, T. L. (1989). Comparable work, occupational labor markets, and occupational earnings: Results from the 1980 Census. In R. Michael, H. Hartmann, \& B. O'Farrell (Eds.), Pay equity: Empirical inquiries (pp. 134-152). Washington, DC: National Academy Press.

Parcel, T. L., \& Menaghan, E. G. (1994). Parents' jobs and children's lives. New York: Aldine de Gruyter.

Perry-Jenkins, M., Repetti, R. L., \& Crouter, A. C. (2000). Work and family in the 1990s. Journal of Marriage and the Family, 62, 981-998.

Peterson, N. G., Mumford, M. D., Borman, W. C., Jeanneret, P. R., Fleishman, E. A., Levin, K. Y., Campion, M. A., Mayfield, M. S., Morgeson, F. P., Pearlman, K., Gowing, M. K., Lancaster, A. R., Silver, M. B., \& Dye, D. M. (2001). Understanding work using the Occupational Information Network (O*Net). Personnel Psychology, 54 , 451-492.

Reskin, B. (1993). Sex segregation in the workplace. Annual Review of Sociology, 19, 241-270. 
Schock, A. M., Gavazzi, S. M., Fristad, M. A., \& Goldberg-Arnold, J. (2002). The role of father participation in the treatment of childhood mood disorders. Family Relations, 51, 230-237.

Tabachnick, B. G., \& Fidell, L. S. (2001). Using multivariate statistics (4th ed.). Needham Heights, MA: Allyn and Bacon.

U.S. Department of Labor. (1991). Dictionary of occupational titles (4th ed.). Washington, DC: U.S. Government Printing Office.

Zhang, Z., \& Snizek, W. E. (2003). Occupation, job characteristics, and the use of alcohol and other drugs. Social Behavior and Personality, 31, $395-503$

Zimmerman, F. J., Christakis, D. A., \& Vander Stoep, A. (2004). Tinker, tailor, soldier, patient: Work attributes and depression disparities among young adults. Social Science and Medicine, 58, 1889-1901.

\section{Appendix}

To use the $\mathrm{O}^{*}$ Net, go to the following online site: http://www.onetcenter.org. The first step is to assign an $\mathrm{O} * \mathrm{Net}$ SOC Code to each occupation in your data set. The O*Net SOC Code is the identifier that links a particular occupation description and the other files on the site that contain information about this particular occupation. To find an $\mathrm{O}^{*} \mathrm{Net}$ SOC code

1. Click on "O*Net Online"

2. Click on "Find Occupations"

3. Enter a key word from the descriptive data you have collected (e.g., teacher)

4. Select the occupation that most closely matches the job description

5. Enter and save (XX-XXXX.xx) the O*Net SOC code into a spreadsheet that also contains your study identifiers.

Once the $\mathrm{O}^{*} \mathrm{Net}$ SOC codes are assigned, the next step is to merge additional variables of interest from the $\mathrm{O}^{*} \mathrm{Net}$ Web site. Some programming ability is needed to do this because it requires rearranging the information found on the Web site. To create a table of $\mathrm{O}^{*} \mathrm{Net}$ values to be merged with our data, we downloaded text files of interest to us from the O*Net Web site and wrote a SAS program to read the required data out of these files into a SAS data set. In our case, each observation in that SAS data set had the O*NET-SOC code (from onetsoc data.txt), the 15 work activity values (from WorkActivity.txt), the 21 context values (from Work Context.txt), and the job zone (from onetsoc_job_ zones.txt) for that SOC code. The SAS program was used to pull values out of the columns in the text files. In our case, we kept information from the O*Net SOC code, Element Name, Scale ID, and Data Value columns in the text files. Having extracted the information of interest from these text files, the final step was to merge this information with the jobs in our sample by $\mathrm{O} * \mathrm{Net}-\mathrm{SOC}$ code. Directions for standardizing these raw values were found on the O*Net Web site at http://www. onetcenter.org/faqDatabase.htm.

To access the $\mathrm{O}^{*}$ Net files

1. While on http://www.onetcenter.org, click on Developers Corner

2. Click on Production Database

3. Go to Database and Dictionary Download

4. Choose the type of program you will use to download the data.

For those who prefer not to work with text files, it is possible to link to the National Crosswalk Service Center where Microsoft Access, VisualFoxPro, or SAS/PC versions of the O*Net 8.0 database are currently available for download. These files, however, also need to be processed to create a table with the O*Net SOC code and the characteristics of that particular job. 